\title{
Mapping the Process and the Resources That Are Needed to Implement a Praziquantel Mass Drug Administration Program for Children Aged Five Years Old and Below in Resource Limited Settings: a Scoping Review
}

Mhlengi Vella Ncube ( $\nabla$ mhlengivncube@yahoo.com )

University of KwaZulu-Natal School of Nursing and Public Health https://orcid.org/0000-0002-83179856

\section{Muhubiri Kabuyaya}

University of KwaZulu-Natal School of Nursing and Public Health

Moses John Chimbari

University of KwaZulu-Natal School of Nursing and Public Health

Research

Keywords: Schistosomiasis, Treatment, Children, Praziquantel, Pre-school

Posted Date: April 1st, 2021

DOl: https://doi.org/10.21203/rs.3.rs-362365/v1

License: (c) (1) This work is licensed under a Creative Commons Attribution 4.0 International License. Read Full License 


\section{Abstract}

Background: The early childhood development of millions of children in some low and medium income countries may be compromised by schistosomiasis infections contracted at the age of 5 years or below. Currently there are no standard guidelines for treating schistosomiasis in children that are five years and younger using praziquantel (PZQ), the only drug that the WHO recommends to treat schistosomiasis. This review is on processes and resources involved in the treatment of schistosomiasis in children aged five years and below.

Methods: An electronic search for peer-reviewed articles published in the period from 2008 to 2018 (August) was done in the Academic Search Complete, CINAHL with Full Text, Health Source:

Nursing/Academic Edition, and MEDLINE databases via EBSCOHost and Google Scholar databases. The search targeted journals that described the treatment of schistosomiasis in children five years and below using praziquantel.

Results: Twelve studies met the inclusion criteria. The process of treating schistosomiasis in the children aged five years old and below included the following activities: enrolment of the children into the treatment program; clinical examination; diagnosis; taking anthropometric measurements; feeding the children, making the PZQ palatable to the children; administration of PZQ and monitoring of side effects. There was also a variation in the resources used to treat children aged five- and below for schistosomiasis.

Conclusions: A PZQ mass drug administration program for children aged five years old and below in endemic areas should exclude diagnosis of schistosomiasis before treatment. The resources required in the treatment process should be affordable, should not require skills and maintenance resources that are beyond those that are available at primary healthcare level.

\section{Background}

Globally, over 240 million people mainly in impoverished communities (4), are affected by schistosomiasis (Mo, Agosti et al. 2014, Ross, Olveda et al. 2014, Olveda, McManus et al. 2016). One hundred and twenty three million (123 M) of people affected by schistosomiasis are children(Mduluza and Mutapi 2017) and among them 23 million are below the age of 6 years. Ninety percent (90\%) of the cases of schistosomiasis are found in Sub-Saharan Africa (WHO 2017, French, Evans et al. 2018). Children infected with schistosomes at the age of five years and below may have compromised development manifesting as stunted growth, lethargy, cognitive and memory impairment (Adenowo, Oyinloye et al. 2015). If children infected by schistosomes at five years and below are not treated, their academic performance may be negatively affected and hence there will be trapped in the poverty cycle in later years of their lives(Adenowo, Oyinloye et al. 2015). Thus, treatment of schistosomiasis in children five years old and below is important to prevent ailment, promote healthy development and fight poverty(Dabo, Bary et al. 2013). 
The World Health Organization recommends the use of Praziquantel (PZQ) to treat schistosomiasis(WHO 2018). PZQ has been used to control schistosomiasis in affected communities through mass drug administration (MDA)(Olveda, McManus et al. 2016). MDA programs have excluded children aged five years and below based on the misconception that this age group is not exposed to schistosomiasis(Stothard, Sousa-Figueiredo et al. 2011) and also because they do not attend school where the programs focus(Osakunor, Mduluza et al. 2018). Some children in communities where schistosomiasis is endemic have been diagnosed for schistosomiasis in their first year of lif e(Stothard, Sousa-Figueiredo et al. 2011). These children remain infected and unwell until the age of six when they start school and are enrolled in school-based treatment programs. Early treatment of these children will prevent morbidity, promote early childhood development and facilitate socio-economic development in affected communities(Dabo, Bary et al. 2013).

The recommended dose of PZQ to treat schistosomiasis is $40 \mathrm{mg} / \mathrm{kg}(\mathrm{WHO} 2018)$. Merk and Bayer, when registering PZQ, overlooked its purpose for the use of the drug to treat children below the age five(Stothard, Sousa-Figueiredo et al. 2013). When the need to treat this age group was realized, the use of the drug on children was done without legal protection, or evidence of safety and efficacy(Stothard, Sousa-Figueiredo et al. 2013). The WHO supported studies to determine the safety and efficacy of PZQ in children five years and below(Stothard, Sousa-Figueiredo et al. 2013). These studies showed that PZQ is safe and efficacious for treating schistosomiasis in children five years old and below (Navaratnam, Sousa-Figueiredo et al. 2012, Garba, Lamine et al. 2013). Thus, WHO has recommended that children aged five years and below in endemic areas be treated at a dose of $40 \mathrm{mg} / \mathrm{kg}$ during preventive MDA programs(WHO 2018). However, there are no specific treatment guidelines for using PZQ in this age group.

Numerous challenges associated with treating schistosomiasis in children aged five years and below have been reported(Stothard, Sousa-Figueiredo et al. 2013). The PZQ tablet is bitter and difficult to swallow for young children because of its size(Osakunor, Woolhouse et al. 2018). The healthcare systems in some of the areas affected by schistosomiasis cannot afford to purchase or maintain scales(Osakunor, Woolhouse et al. 2018). In the absence of scales, WHO recommends the use of height based dose poles to determine PZQ doses(Sousa-Figueiredo, Betson et al. 2012). The WHO approved dose pole does not cater for children who are less than $60 \mathrm{~cm}$ tall(Sousa-Figueiredo, Betson et al. 2012). Children with stunted growth, who are in need of treatment, may fall outside the scale of the dose pole. PZQ also causes minor side effects that require close monitoring(Bayer 2010). Any treatment process that is developed needs to consider these challenges.

We reviewed the processes followed in the use of PZQ to treat schistosomiasis in children aged five years and below. We reported on: the PZQ treatment process and resources that were used to treat schistosomiasis in children aged five years old and below different clinical studies; and the process followed and resources that could be used in the implementation of a PZQ MDA program targeting children under five. MDA programs for children are essential in promoting early childhood development and to contribute to ending poverty(Dabo, Bary et al. 2013). 


\section{Methods}

We used the scoping review methodology for this study because scoping reviews are recommended when the purpose of the study is "to examine how research is conducted on a certain topic or field."(Munn, Peters et al. 2018) The Arksey and O'Malley's framework for scoping reviews with modifications from Levacet al (Arksey and O'Malley 2005, Levac, Colquhoun et al. 2010) was used to describe the process and resources involved in treating schistosomiasis in children below 5 years old. However, we did not conduct the interviews outlined in the Arksey and O'Malley's framework (2010) methodology.

The research question for this review was: "what are the processes and resources that are required to treat schistosomiasis in children below the age of five years? "

The search terms schistosomiasis, bilharzia, treatment, Praziquantel and under five years old, pre-school children were used to find studies from the Google Scholar database. The following combination of search words and Boolean terms were used: Schistosomiasis OR bilharzia AND treatment OR praziquantel AND children under 5 years old to search for studies from the EBSCOHost database. The EBSCOHost Databases that were searched are: Academic Search Complete, CINAHL with Full Text, Health Source: Nursing/Academic Edition and MEDLINE.

The searches covered a ten-year period; January 2008 to August 2018. Only peer reviewed journal articles reporting on primary data were included in the review. Peer reviewed journal articles reporting on treatment of schistosomiasis in children aged five years and below using drugs other than praziquantel were excluded because PZQ is the only drug that has been approved by WHO for use in schistosomiasis treatment programs.

Studies, reporting on the use of Epiquantel were, however included because PZQ is the active ingredient in the Epiquantel syrup. Peer reviewed journal articles not written in English were excluded because we did not have translation resources. The searches were initially saved on libraries created in the EBSCOHost and Google Scholar databases. Completed searches were merged on to Endnote X10. EBSCOHost removed several duplicates during the transfer process. The remaining duplicates were removed using ENDNote.

The scope of the review was limited to studies reporting on the technical activities involved in the treatment of schistosomiasis of the species $S$. hematobium and S. mansoni in children aged five and below. We thus included all studies that reported on the use of praziquantel to treat schistosomiasis in children aged five years old and below. Studies that reported on treatment of schistosomiasis in children above five years were included if the studies also reported on treatment of under-five years old as a category. Since it is not necessary to diagnose schistosomiasis during a PZQ MDA program(WHO 2006), studies reporting exclusively on the diagnosis of schistosomiasis (and did not include treatment of under children aged five years old and below in the methodology) were excluded. Title, abstract and full article screening were used to identify articles that met our inclusion criteria 
A modified Donabedian framework was used to analyse the findings in our study(Donabedian 2005). The Donabedian framework separates operations into structure (resources), processes and outcomes(Donabedian 2005). We identified the processes and resources involved in the treatment of schistosomiasis in children under five years old. We then used the processes that were reported in the studies as a framework on which we constructed a treatment process that can be used to implement a schistosomiasis control MDA program for children under five years old. We also used the resources identified in the study to select those that would be appropriate for use in a schistosomiasis control MDA program for children aged five years and below.

A PRISMA checklist was used to assess the manuscript(Moher, Liberati et al. 2010).

\section{Results}

Our initial search yielded 1088 titles. After removing duplicates, titles reporting exclusively on children older than five years, review papers and studies that focused on diagnosis remained with 35 studies. Twenty-three (23) of those studies were removed after full article screening because they were focused on diagnosis or did not describe the processes, materials and outcomes that were used in the treatment process. Twelve studies met the search criteria (Fig. 1).

Twelve peer reviewed articles met our inclusion criteria (table 1). The studies in these articles were from five different countries: Ghana (41.7\%), Ivory Coast (16.7\%), Niger (8.3\%), Sudan (8.3\%) and Zimbabwe (16.7\%). The lowest age of the children reported in the studies was 1 month old and the highest age reported was 93 months. Ten of the studies reported on the use of the praziquantel (PZQ) tablet, 1 study reported on the use of PZQ syrup and one study reported on the use of both the PZQ tablet and the PZQ syrup.

\section{Outcomes}

All the studies that we reviewed reported that praziquantel is efficacious against schistosomiasis in children under five years. The lowest PZQ dose that was efficacious against schistosomiasis in this age group was $20 \mathrm{mg} / \mathrm{kg}$ (Coulibaly, Panic et al. 2017, Coulibaly, Panic et al. 2018). All the studies reported on the PZQ dose of $40 \mathrm{mg} / \mathrm{kg}$ to treat schistosomiasis in this age group. The difference in PZQ efficacy observed between treatment of children aged five years old and below with a PZQ dose of $40 \mathrm{mg} / \mathrm{kg}$ and that of $60 \mathrm{mg} / \mathrm{kg}$ was insignificant(Mutapi, Rujeni et al. 2011).

All the studies we reviewed reported PZQ to be safe for use to treat schistosomiasis in children aged five years old and below. The highest safe dose of PZQ reported was $60 \mathrm{mg} / \mathrm{kg}$ (Coulibaly, Panic et al. 2017, Coulibaly, Panic et al. 2018). All the studies reported that there were no serious adverse events reported after treating schistosomiasis in children aged five years and below with PZQ doses of between $20 \mathrm{mg} / \mathrm{kg}$ to $60 \mathrm{mg} / \mathrm{kg}$. We recommend that the WHO recommended dose of $40 \mathrm{mg} / \mathrm{kg}$ should be used to treat schistosomiasis during schistosomiasis control MDA programs for children aged five years old and below years old. 


\section{Process}

All the studies followed the same treatment sequence: enrolment of children into the program; clinical examination; diagnosis; weight and height measurements; treatment and the monitoring of side effects. Enrolment involved acquiring consent from parents and recording all the children that participated in the study. The enrolled children were subjected to clinical examination to make sure that the children were safe to treat. Based on the clinical examinations the children who were considered safe for treatment were those who: were generally well(Amin, Swar et al. 2012, Coulibaly, N'Gbesso et al. 2012, Navaratnam, Sousa-Figueiredo et al. 2012, Sousa-Figueiredo, Betson et al. 2012, Garba, Lamine et al. 2013, Wami, Nausch et al. 2016); had had no recent illness(Wami, Nausch et al. 2016); were not suffering from or receiving treatment for tuberculosis (TB)(Wami, Nausch et al. 2016); did not have malaria(Navaratnam, Sousa-Figueiredo et al. 2012); had not undergone a major surgical procedure(Wami, Nausch et al. 2016); were not suffering from a fever(Namwanje, Kabatereine et al. 2011, Wami, Nausch et al. 2016) and did not have a history of adverse drug reactions(Namwanje, Kabatereine et al. 2011). The appropriate interventions were provided to the enrolled children who were illegible for PZQ treatment on the basis of ill health.

Diagnosis of schistosomiasis was done in all the studies to determine baseline prevalence and intensity in the children before treatment. Weight and height(Sousa-Figueiredo, Pleasant et al. 2010, SousaFigueiredo, Betson et al. 2012, Coulibaly, Panic et al. 2017) measurements were then done to determine the required dose of $P Z Q$ to treat children. Administration of $P Z Q$ included feeding the children to reduce side effects(Nalugwa, Nuwaha et al. 2015) and to increase the assimilation of PZQ(Sousa-Figueiredo, Betson et al. 2012, Garba, Lamine et al. 2013); making the PZQ palatable for the children by breaking or crushing the tablet and mixing it with a sweeter; and feeding the tablets to the children. After orally taking in the tablet, the children were monitored for side-effects. The most immediate side effect that was observed for was vomiting the tablet within the first 20-60minutes(Mutapi, Rujeni et al. 2011, Coulibaly, N'Gbesso et al. 2012), in which case the tablet was re-administered to the children. Other side-effects were recorded and appropriate treatment was provided to the children.

Using the processes carried out in the 12 clinical studies to treat schistosomiasis in children aged five years and below, we designed a treatment process that can be used to implement a schistosomiasis mass drug administration (MDA) program for this age group (Fig. 2). In the MDA treatment process diagnosis of schistosomiasis could be done only on a sample of the children before the clinical examination is done.

\section{Structure}

The materials that were used to treat schistosomiasis in children under five years old in the studies are listed in table 2. In the same table we have proposed materials that could be used in a schistosomiasis MDA program for children under five years old in resource limited settings. Registers were used to enroll 
children into the clinical studies. For a schistosomiasis control MDA program for children under five we recommend electronic registers that are embedded in electronic tablets. The Kato-Katz and schistosomiasis urine filtration kits(Garba, Barkiré et al. 2010, Mutapi, Rujeni et al. 2011, Amin, Swar et al. 2012, Coulibaly, N'Gbesso et al. 2012, Nalugwa, Nuwaha et al. 2015, Wami, Nausch et al. 2016, Coulibaly, Panic et al. 2017, Coulibaly, Panic et al. 2018) were the most used materials to diagnose Schistosomiasis mansoni and Schistosomiasis haematobium respectively in the studies. The stools and urine for diagnosis were collected in specimen containers(Namwanje, Kabatereine et al. 2011). Serological tests: Point-of-care circulating cathodic antigen (POC-CCA)(Sousa-Figueiredo, Pleasant et al. 2010, Coulibaly, N'Gbesso et al. 2012, Coulibaly, Panic et al. 2017); soluble egg antigen enzyme-linked immunosorbent assay SEA-ELISA(Sousa-Figueiredo, Pleasant et al. 2010); microalbumin reagent strips(Wami, Nausch et al. 2016) and urinalysis dip sticks(Wami, Nausch et al. 2016) were also used to test for schistosomiasis in the children. Questionnaires were also used for diagnosis(Sousa-Figueiredo, Pleasant et al. 2010, Mutapi, Rujeni et al. 2011, Amin, Swar et al. 2012, Sousa-Figueiredo, Betson et al. 2012, Garba, Lamine et al. 2013, Wami, Nausch et al. 2016, Coulibaly, Panic et al. 2017, Mduluza and Mutapi 2017, Coulibaly, Panic et al. 2018). We recommend the use of questionnaires and urine dipsticks for the diagnosis of urinary schistosomiasis and the POC-CCA for the diagnosis of intestinal schistosomiasis on the field for use in schistosomiasis control MDA programs for children aged under five years. Questionnaires and the child health booklet(Garba, Lamine et al. 2013) were used for clinical assessments. We also recommend that both be used for clinical assessments in the schistosomiasis control MDA programs for children aged under five years old and below years old. Some the studies that we reviewed reported using weight scales, while others used stadiometers (Sousa-Figueiredo, Pleasant et al. 2010, Amin, Swar et al. 2012) and one used dose poles(Sousa-Figueiredo, Pleasant et al. 2010) to obtain the anthropometric measurement that were used to calculate the dose amount of PZQ. We recommend the use of the dose pole and/or tape measures to make these measurements in schistosomiasis control MDA programs for young children.

Bread and juice(Mutapi, Rujeni et al. 2011, Navaratnam, Sousa-Figueiredo et al. 2012, Sousa-Figueiredo, Betson et al. 2012, Nalugwa, Nuwaha et al. 2015, Wami, Nausch et al. 2016, Coulibaly, Panic et al. 2017), millet wafer(Garba, Lamine et al. 2013) and porridge(Garba, Lamine et al. 2013) were the food items that were used in the studies we reviewed. PZQ tablets were crushed with spoons(Mutapi, Rujeni et al. 2011) or pestle and mortar(Coulibaly, Panic et al. 2017, Coulibaly, Panic et al. 2018) to make them small enough for the children to take in. The studies used honey and sugar(Amin, Swar et al. 2012), juice and syrup flavored water as sweeteners(Mutapi, Rujeni et al. 2011, Navaratnam, Sousa-Figueiredo et al. 2012, Sousa-Figueiredo, Betson et al. 2012, Nalugwa, Nuwaha et al. 2015, Wami, Nausch et al. 2016, Coulibaly, Panic et al. 2017) to mask the bitter taste of PZQ. We recommend that children aged five years old and below should be fed with bread and juice or instant porridge during schistosomiasis control MDA programs for this age group. We recommend the use of tablets that were crushed with a pestle and mortar and juice as the sweetener for use in these MDA programs.

Some of the studies reported using schools(Mutapi, Rujeni et al. 2011, Namwanje, Kabatereine et al. 2011, Wami, Nausch et al. 2016), early childhood development (ECD) centers(Wami, Nausch et al. 2016), 
healthcare centers(Navaratnam, Sousa-Figueiredo et al. 2012), clinics(Sousa-Figueiredo, Pleasant et al. 2010) and churches(Navaratnam, Sousa-Figueiredo et al. 2012) as sites where the children were recruited. The schools were used as treatment centers in studies that involved comparisons between children that were aged five years old and below (of pre -school aged children (PSAC)) and school aged children (SAC)(Wami, Nausch et al. 2016). The ECD centers that are reported on in the studies were attached to the schools were the SAC participants were found(Wami, Nausch et al. 2016). We recommend the use of schools and clinics as treatment centers for schistosomiasis control MDA programs for children aged five years old and below.

The human resources that took part in the clinical aspects of the studies were from a variety of healthcare professions. These medical staff included pediatricians(Namwanje, Kabatereine et al. 2011), medical doctors(Garba, Lamine et al. 2013), nurses(Mutapi, Rujeni et al. 2011) and laboratory technicians(Coulibaly, Panic et al. 2017, Coulibaly, Panic et al. 2018). Some studies did not specify the professions of the clinical human resources only indicating that medical staff, clinicians or health officers were part of the study. One study stated the involvement of community leaders and community drug distributors(Sousa-Figueiredo, Pleasant et al. 2010) as part of their human resources. We recommend nurses to be the human healthcare professionals that implement the schistosomiasis control MDA programs for children aged five years old and below.

\section{Challenges}

Some of the studies reported the use of the current WHO approved 600mg PZQ dose tablet formulation as a challenge when treating schistosomiasis in children aged five years old and below, especially when implementing a large scale treatment program(Davis and Schul 1993, Nalugwa, Nuwaha et al. 2015, Coulibaly, Panic et al. 2017). They recommended that a variation of this formulation with three partitions that make it possible to split the tablet into four pieces of $150 \mathrm{mg}$ each should be used when treating schistosomiasis in children aged five years old and below so as to ensure the use of the correct PZQ to treat schistosomiasis in these children(Sousa-Figueiredo, Pleasant et al. 2010). The studies proposed that a pediatric formulation should be developed and that the PZQ pediatric formulations could be an orally dispensable tablet(Coulibaly, Panic et al. 2017) or a PZQ syrup(Garba, Lamine et al. 2013).

\section{Discussion}

The objective of this study was to map the processes, and resources that are required to implement a schistosomiasis control MDA program for children aged five years old and below. A schistosomiasis control MDA program for children aged five years old and below years old has not been reported on. The closest to a schistosomiasis MDA program for this age group is mainly clinical studies that were recommended by WHO to determine the safety and efficacy of treating schistosomiasis in children aged five years old and below using praziquantel(Stothard, Sousa-Figueiredo et al. 2013). We modified the 
processes that were used in these studies to propose a treatment process to execute a schistosomiasis control MDA program for children aged five years and below.

\section{Process}

The praziquantel treatment process used in an MDA program for children aged five years old and below could follow the sequence: enrolment of children; clinical examination; weight and height measurements; treatment and the monitoring of side effects for all the children. This is in line with recommendations of the WHO that individual diagnosis of schistosomiasis prior to treatment is not required before treatment during an MDA program(WHO 2006). The studies we reviewed diagnosed all the children in order to determine the prevalence of schistosomiasis in the children that are aged five years and below and also to determine the efficacy of PZQ in treating schistosomiasis in these children. The diagnosis of schistosomiasis in the MDA program is important to determine the baseline prevalence and burden of infectivity of schistosomiasis in the children for the purposes of monitoring and evaluation(Olveda, Acosta et al. 2016). Diagnosis of schistosomiasis in a schistosomiasis control MDA program for children aged five years old and below for monitoring and evaluation could be done on a random sample of the children soon after they have been enrolled into the treatment program(Martin, Bid et al. 2015). In some cases, the baseline prevalence and infection intensity could be done separately from the MDA program during disease surveillance programs making the treatment process to move directly from enrolment of the children to clinical assessments(Health 2019). The disease surveillance programs when present could be used to monitor and evaluate the schistosomiasis control MDA program for children aged five years and below.

\section{Structure}

The Donabedian framework refers to resources as structural components of a healthcare system(Donabedian 2005). The resources that are required to implement a schistosomiasis control MDA program for children aged five years old and below should be applicable to the environment in which the MDA program will be implemented(Betson, Sousa-Figueiredo et al. 2010). Most of the places where schistosomiasis is endemic are economically disadvantaged and therefor suffer from resource constraints. We used relative cost and ease of access to select the resources that we recommended to be the best for use in a schistosomiasis control MDA program for children aged five years and below. The resources used should also be eco-friendly(Avinash, Avinash et al. 2013).

None of the studies described the nature of the registers that they used for enrolment. Electronic registers that are embedded in tablets could be used to enrol children into a schistosomiasis control MDA program. The use of electronic information management systems in the MDA program could be extended to storing and analysing: the clinical assessments of the children to identify other childhood illnesses that need mass intervention. Tablets can be used for controlling the quality of the medical information about the children that is requested using questionnaires; for managing and monitoring the program's consumables inventory and for monitoring and evaluation of the progress and impact of the MDA program. A multinational study conducted in low and medium income countries (Ghana, Kenya, India and 
Pakistan) reported that the initial cost of purchasing electronic tablets and software licensing costs for healthcare program data management could be high(Dickinson, McCauley et al. 2019). However, because the tablets could be used multiple times in the same program and also across different programs, the purchase of tablets could be a justifiable investment when taking into account economies of scale and economies of scope in healthcare program implementation cost analysis(Turner, Toor et al. 2017, Dickinson, McCauley et al. 2019). The limitations of using electronic tablets could include intermittent electricity supply and internet coverage in some of the areas where the schistosomiasis control MDA program could be necessary(King, Hall et al. 2014, Dickinson, McCauley et al. 2019). The use of paper based registers is most common and carries some hidden costs(Dickinson, McCauley et al. 2019). When the initial cost of buying and setting up electronic systems for treatment data management cannot be afforded, paper based registers could be used.

The WHO recommends that diagnosis should not be made a requirement for the mass drug administration of praziquantel in schistosomiasis endemic areas(WHO 2006). Diagnosis could however be used for the purposes of monitoring and evaluation of a schistosomiasis control MDA program. To diagnose schistosomiasis in children aged five years old and below for the purposes of monitoring and evaluating a schistosomiasis control MDA program targeting this age group, urine deep stick to detect haematuria caused by urinary schistosomiasis and the POC-CCA to detect intestinal schistosomiasis could be used. Both these methods have the advantages of being performed at the site of the MDA program and are more sensitive that the microscopy based methods that are normally used to diagnose schistosomiasis in school going children(Betson, Sousa-Figueiredo et al. 2010, Ochodo, Spek et al. 2012, Sousa-Figueiredo, Betson et al. 2013). The urinary dipstick and POC-CCA point of care tests do not require specialised human resources, in particular laboratory technicians, to carry out the testing. Other methods such as the PCR and the FLOTAC technologies require additional specially skilled technicians and equipment such as thermocyclers and centrifuges which cannot be used on the field(Utzinger, Becker et al. 2015).

The activities that are involved in treating schistosomiasis in children aged five years and below include taking weight or height measurements; calculating dosage; feeding the children; making PZQ more palatable to the children and administering the PZQ to the children. Height rather than weight could be used to determine the dose of PZQ to be given to the children(Sousa-Figueiredo, Betson et al. 2012). This is because the dose poles that are used to measure height are less expensive to buy and to maintain compared to the weight scales that could be used in the event that weight is used to determine dosage(Mutapi 2015). The extended dose pole by Sousa-Figueiredo et al,2012 addresses the limitation of the exclusion of children shorter than $94 \mathrm{~cm}$ from being treated based on the dose pole(Sousa-Figueiredo, Betson et al. 2012). The use of electronic health systems management could eliminate the need for dose poles and weight scales by providing data that could be used to perform weight-for-age calculations(Olliaro, Vaillant et al. 2013). The age of the child could then be used to calculate the appropriate PZQ dose to treat the children(Olliaro, Vaillant et al. 2013). 
We recommend that bread and juice are the most suitable foods to feed the children before treatment. This is because bread and juice are filling and yet easily available in most communities and also require very little labour to prepare(Leyvraz, Mizéhoun-Adissoda et al. 2018). Fortified spreads could be used on the bread to enhance the nutritional value of the bread(Matilsky, Maleta et al. 2009). Juice also has the added capability to sweeten the PZQ making it more palatable for the children(Bunupuradah, Wannachai et al. 2006). An alternative to bread and juice could be fortified cereal(Bulusu, Laviolette et al. 2007). Fortified has the advantage of providing multi-nutrients to children(Bulusu, Laviolette et al. 2007) thereby addressing the needs of the schistosomiasis control MDA program for children aged five years old and below and also tackling the challenge of malnutrition(Frigerio, Macario et al. 2016). Schistosomiasis and childhood often exist as co-epidemics(Frigerio, Macario et al. 2016, Osakunor, Woolhouse et al. 2018). The studies reported using either pestle and mortar or spoons to crush the PZQ tablets. Ideally the pestle and mortar could be used. This is because the pestle and mortar are the best tools to use to crush the tablets and are also inexpensive(Thong, Manrique et al. 2018). Spoons are less efficient at crushing tablets but could also be used when pestle and mortar are not available.

Schistosomiasis control MDA programs for children aged five years old and below could be carried out in clinics or schools. The use of clinics for such an MDA program is encouraged by the WHO(WHO 2018). Schools on the other hand have been successfully used by schistosomiasis control MDA programs to treat SAC(Mutapi, Rujeni et al. 2011, Burnim, Ivy et al. 2017). Since some ECD centres are attached to schools, the same operational strategies that have been used to target SAC can be used to target PSAC(Mutapi, Rujeni et al. 2011, Wami, Nausch et al. 2016). Children who are not enrolled in ECD centres can be invited to be part of the MDA program. Faith-based establishments such as churches and mosques are used for healthcare programs in some resource limited settings(Berra, Franklin et al. 2017, Blevins, Kiser et al. 2017) and could be used to mobilize people to enrol their under five children in the schistosomiasis control MDA program (Peterson, Atwood et al. 2002, King, Warsi et al. 2017). Faith-based establishments could also be used as treatment sites for the MDA program. The choice between using clinics, ECD centres, crèches and faith-based establishments depends on the prevailing government policy and on where the children under five years who are at risk of schistosomiasis could be best accessed for treatment in the community where the MDA program is required.

The studies we reviewed reported on using a variety of human resources such as paediatricians, medical doctors, nurses, laboratory technicians and community health works. Paediatricians, medical doctors and laboratory technicians are seldom available in resource limited settings such as those that are affected by schistosomiasis(Coulibaly, Cavalli et al. 2008). Nurses could therefore implement the schistosomiasis control MDA program for children aged five years old and below. This is because nurses have been found to successfully implement paediatric healthcare interventions at primary healthcare level in the resource limited setting that schistosomiasis is mostly found(Janssen, Ndirangu et al. 2010). Due to human resources constrains in most areas affected by schistosomiasis community health workers are needed to support the clinicians in MDA programs(Nandha, Sadanandane et al. 2007, Dean, Page et al. 2016). Considerations of the disruption of routine curative functions of the nurses in the clinics where the nurses normally work and the remuneration motivation of community care givers should be taken into account 
when developing a human resources strategy for the implementation of an MDA program(Coulibaly, Cavalli et al. 2008, Hodges, Sonnie et al. 2012) to control schistosomiasis in children aged five years old and below.

The strengths of this study are that the studies are from five different countries which makes them generalizable to resource limited settings in Africa and similar settings globally. The limitations of the study are that we did not perform interviews as recommended by Arksey and O'Malley's framework for systematic reviews(Arksey and O'Malley 2005). The interviews would have given us more information on the resources that are applicable for use in schistosomiasis control MDA programs for children under five years in different settings. We, however could not perform these interviews due to challenges in getting authorization from several countries to conduct the interviews within the duration of the study.

\section{Conclusion}

The process that could be followed in the implementation of a schistosomiasis control MDA program for children aged five years old and below is: enrolment of children into the treatment program; clinical examination; weight and height measurements; treatment and the monitoring of side effects for all the children (Fig. 2). Diagnosis could be done for the purpose of monitoring and evaluation either before the MDA program or immediately after the enrolment stage of the MDA program. The resources that could be used for the treatment program are: electronic tablets; urine dipsticks; POC-CCA; child health booklet; questionnaire; dose pole; tape measures; bread, juice, pestle and mortar; spoons; PZQ tablets; clinics and ECD centres. The availability of these resources in different healthcare centres may vary resulting in the

need for unavailable resources to be purchased prior to the program. Resources that are already available and routinely in use at primary healthcare care level should be prioritized for use in the MDA program.

\section{Abbreviations}




\begin{tabular}{|ll|}
\hline ALB: & Albendazole \\
\hline ECD: & Early Childhood Development \\
\hline HAART: & highly active antiretroviral therapy \\
\hline MDA & Mass Drug Administration \\
\hline MEDLINE: & Medical Literature Analysis and Retrieval System Online \\
\hline POC CCA & Point-of-care Circulating Cathodic Antigen \\
\hline PSAC & Pre-School Aged Children \\
\hline PZQ: & Praziquantel \\
\hline SAC & School Aged Children \\
\hline SEA-ELISA & Soluble Egg Antigen Enzyme-Linked Immunosorbent Assay \\
\hline STH: & Soil Transmitted Helminths \\
\hline TB: & Tuberculosis \\
\hline UKZN: & University of KwaZulu-Natal \\
\hline WHO: & World Health Organisation \\
\hline
\end{tabular}

\section{Declarations}

\section{Acknowledgements}

The authors are grateful for the support provided by Dr Elizabeth Ojewole and Dr Resign Gunda in reviewing the manuscript. The authors would like to acknowledge the College of Health Sciences (CHS) through CHS scholarship program at the University of KwaZulu-Natal for their financial support. This research was commissioned by the National Institute of Health Research using Official Development Assistance (ODA) funding. The views expressed in this publication are those of the author(s) and not necessarily those of the NHS, the National Institute of Health Research, or the Department of Health.

\section{Availability of data and materials}

Data will be made available upon request

\section{Ethics approval and consent to participate}

Not applicable.

\section{Funding}

University of KwaZulu-Natal 
NIHR Global Health Research Unit Tackling Infections To Benefit Africa

British Academy's Early Childhood Development Program

\section{Authors' contributions}

MVN conceived the in collaboration with CM and MC. MVN designed the search strategy. MV performed searches. MVN and KM conducted data selection and extraction. All authors were involved in data analysis and interpretation of results. All authors revised and approved the final manuscript.

\section{Consent for publication}

All authors consent and approve the manuscript for publication.

\section{Competing interests}

The authors declare no that they have no competing interests.

\section{Ethics approval and consent to participate}

Not applicable.

\section{References}

1. Adenowo, A. F., B. E. Oyinloye, B. I. Ogunyinka and A. P. Kappo (2015). "Impact of human schistosomiasis in sub-Saharan Africa." Brazilian Journal of Infectious Diseases 19: 196-205.

2. Amin, M., M. Swar, M. Kardaman, D. Elhussein, G. Nouman, A. Mahmoud, A. Appiah, A. Babiker and M. Homeida (2012). "Treatment of pre-school children under 6 years of age for schistosomiasis: safety, efficacy and acceptability of praziquantel." Sudan Journal of Medical Sciences 7(2): 67-76.

3. Arksey, H. and L. O'Malley (2005). "Scoping studies: towards a methodological framework." International Journal of Social Research Methodology 8(1): 19-32.

4. Avinash, B., B. Avinash, B. Shivalinga, S. Jyothikiran and M. Padmini (2013). "Going green with ecofriendly dentistry." The journal of contemporary dental practice 14(4): 766.

5. Bayer. (2010). "Batricide (Praziquantel) Fact scheet." from https://www.accessdata.fda.gov/drugsatfda_docs/label/2010/018714s012lbl.pdf.

6. Berra, K., B. Franklin and C. Jennings (2017). "Community-Based Healthy Living Interventions." Progress in Cardiovascular Diseases 59(5): 430-439.

7. Betson, M., J. C. Sousa-Figueiredo, C. Rowell, N. B. Kabatereine and J. R. Stothard (2010). "Intestinal schistosomiasis in mothers and young children in Uganda: investigation of field-applicable markers of bowel morbidity." The American journal of tropical medicine and hygiene 83(5): 1048-1055.

8. Blevins, J., M. Kiser, E. Lemon and A. Kone (2017). "The percentage of HIV treatment and prevention services in Kenya provided by faith-based health providers." Development in Practice 27(5): 646-657. 
9. Bulusu, S., L. Laviolette, V. Mannar and V. Reddy (2007). Cereal fortification programs in developing countries. Issues in Complementary Feeding, Karger Publishers. 60: 91-105.

10. Bunupuradah, T., S. Wannachai, A. Chuamchaitrakool, J. Intasan, T. Nuchapong, W. Neiss, K. Kramm, C. Pancharoen, D. Burger and J. Ananworanich (2006). "Use of taste-masking product, FLAVORx, to assist Thai children to ingest generic antiretrovirals." AIDS Research and Therapy 3(1): 30.

11. Burnim, M., J. A. Ivy and C. H. King (2017). "Systematic review of community-based, school-based, and combined delivery modes for reaching school-aged children in mass drug administration programs for schistosomiasis." PLoS neglected tropical diseases 11(10): e0006043.

12. Coulibaly, J. T., Y. K. N'Gbesso, S. Knopp, J. Keiser, E. K. N'Goran and J. Utzinger (2012). "Efficacy and safety of praziquantel in preschool-aged children in an area co-endemic for Schistosoma mansoni and S. haematobium." PLoS neglected tropical diseases 6(12): e1917.

13. Coulibaly, J. T., G. Panic, K. D. Silué, J. Kovač, J. Hattendorf and J. Keiser (2017). "Efficacy and safety of praziquantel in preschool-aged and school-aged children infected with Schistosoma mansoni: a randomised controlled, parallel-group, dose-ranging, phase 2 trial." The Lancet Global Health 5(7): e688-e698.

14. Coulibaly, J. T., G. Panic, R. B. Yapi, J. Kovač, B. Barda, Y. K. N’Gbesso, J. Hattendorf and J. Keiser (2018). "Efficacy and safety of ascending doses of praziquantel against Schistosoma haematobium infection in preschool-aged and school-aged children: a single-blind randomised controlled trial." BMC medicine 16(1): 81.

15. Coulibaly, Y., A. Cavalli, M. Van Dormael, K. Polman and G. Kegels (2008). "Programme activities: a major burden for district health systems?" Tropical Medicine \& International Health 13(12): 14301432.

16. Dabo, A., B. Bary, B. Kouriba, O. Sankaré and O. Doumbo (2013). "Factors associated with coverage of praziquantel for schistosomiasis control in the community-direct intervention (CDI) approach in Mali (West Africa)." Infectious Diseases of Poverty 2(1): 11.

17. Davis, P. S. and P. L. Schul (1993). "Addressing the contingent effects of business unit strategic orientation on relationships between organizational context and business unit performance." Journal of Business Research 27(3): 183-200.

18. Dean, L., S. Page, K. Hawkins, R. Stothard, R. Thomson, S. Wanji, M. Gyapong, I. Anagbogu, D. Molyneux and S. Theobald (2016). "Tailoring mass drug administration to context: implementation research is critical in achieving equitable progress in the control and elimination of helminth neglected tropical diseases in sub-Saharan Africa." International health 8(4): 233-234.

19. Dickinson, F. M., M. McCauley, B. Madaj and N. van den Broek (2019). "Using electronic tablets for data collection for healthcare service and maternal health assessments in low resource settings: lessons learnt." BMC Health Services Research 19(1): 336.

20. Donabedian, A. (2005). "Evaluating the quality of medical care." The Milbank Quarterly 83(4): 691729. 
21. French, M. D., D. Evans, F. M. Fleming, W. E. Secor, N.-K. Biritwum, S. J. Brooker, A. Bustinduy, A. Gouvras, N. Kabatereine, C. H. King, M. Rebollo Polo, J. Reinhard-Rupp, D. Rollinson, L.-A. Tchuem Tchuenté, J. Utzinger, J. Waltz and Y. Zhang (2018). "Schistosomiasis in Africa: Improving strategies for long-term and sustainable morbidity control." PLOS Neglected Tropical Diseases 12(6): e0006484.

22. Frigerio, S., M. Macario, G. lacovone, K. J. Dussey-Comlavi, P. Narcisi, A. T. Ndiaye, S. Moramarco, R. Alvaro, L. Palombi and E. Buonomo (2016). "Schistosoma haematobium infection, health and nutritional status in school-age children in a rural setting in Northern Senegal." Minerva pediatrica 68(4): 282-287.

23. Garba, A., N. Barkiré, A. Djibo, M. S. Lamine, B. Sofo, A. N. Gouvras, E. Bosqué-Oliva, J. P. Webster, J. R. Stothard, J. Utzinger and A. Fenwick (2010). "Schistosomiasis in infants and preschool-aged children: Infection in a single Schistosoma haematobium and a mixed S. haematobium-S. mansoni foci of Niger." Acta Tropica 115(3): 212-219.

24. Garba, A., M. S. Lamine, A. Djibo, A. Tahirou, M. A. Aouami, A. Alfari, A. E. Phillips, A. Fenwick and J. Utzinger (2013). "Safety and efficacy of praziquantel syrup (Epiquantel®) against Schistosoma haematobium and Schistosoma mansoni in preschool-aged children in Niger." Acta tropica 128(2): 318-325.

25. Health, D. o. (2019). South Africa National Master Plan for the Elimination of Neglected Tropical Diseases (2019 - 2025), South African Department of Health.

26. Hodges, M. H., M. Sonnie, H. Turay, A. Conteh, F. MacCarthy and S. Sesay (2012). "Maintaining effective mass drug administration for lymphatic filariasis through in-process monitoring in Sierra Leone." Parasites \& Vectors 5(1): 232.

27. Janssen, N., J. Ndirangu, M.-L. Newell and R. Bland (2010). "Successful paediatric HIV treatment in rural primary care in Africa." Archives of Disease in Childhood 95(6): 414-421.

28. King, C., J. Hall, M. Banda, J. Beard, J. Bird, P. Kazembe and E. Fottrell (2014). "Electronic data capture in a rural African setting: evaluating experiences with different systems in Malawi." Global health action $7(1): 25878$.

29. King, R., S. Warsi, A. Amos, S. Shah, G. Mir, A. Sheikh and K. Siddiqi (2017). "Involving mosques in health promotion programmes: a qualitative exploration of the MCLASS intervention on smoking in the home." Health Education Research 32(4): 293-305.

30. Levac, D., H. Colquhoun and K. K. O'Brien (2010). "Scoping studies: advancing the methodology." Implementation Science : IS 5: 69-69.

31. Leyvraz, M., C. Mizéhoun-Adissoda, D. Houinato, N. Moussa Baldé, A. Damasceno, B. Viswanathan, M. Amyunzu-Nyamongo, J. Owuor, A. Chiolero and P. Bovet (2018). "Food consumption, knowledge, attitudes, and practices related to salt in urban areas in five sub-saharan African countries." nutrients 10(8): 1028.

32. Martin, D. L., R. Bid, F. Sandi, E. B. Goodhew, P. A. Massae, A. Lasway, H. Philippin, W. Makupa, S. Molina and M. J. Holland (2015). "Serology for trachoma surveillance after cessation of mass drug 
administration." PLoS neglected tropical diseases 9(2): e0003555.

33. Matilsky, D. K., K. Maleta, T. Castleman and M. J. Manary (2009). "Supplementary Feeding with Fortified Spreads Results in Higher Recovery Rates Than with a Corn/Soy Blend in Moderately Wasted Children." The Journal of Nutrition 139(4): 773-778.

34. Mduluza, T. and F. Mutapi (2017). "Putting the treatment of paediatric schistosomiasis into context." Infectious Diseases of Poverty 6: 85.

35. Mo, A. X., J. M. Agosti, J. L. Walson, B. F. Hall and L. Gordon (2014). "Schistosomiasis elimination strategies and potential role of a vaccine in achieving global health goals." The American journal of tropical medicine and hygiene 90(1): 54-60.

36. Moher, D., A. Liberati, J. Tetzlaff and D. G. Altman (2010). "Preferred reporting items for systematic reviews and meta-analyses: the PRISMA statement." Int J Surg 8(5): 336-341.

37. Munn, Z., M. D. J. Peters, C. Stern, C. Tufanaru, A. McArthur and E. Aromataris (2018). "Systematic review or scoping review? Guidance for authors when choosing between a systematic or scoping review approach." BMC medical research methodology 18(1): 143-143.

38. Mutapi, F. (2015). "Changing policy and practice in the control of pediatric schistosomiasis." Pediatrics 135(3): 536-544.

39. Mutapi, F., N. Rujeni, C. Bourke, K. Mitchell, L. Appleby, N. Nausch, N. Midzi and T. Mduluza (2011). "Schistosoma haematobium Treatment in 1-5 Year Old Children: Safety and Efficacy of the Antihelminthic Drug Praziquantel." PLoS Neglected Tropical Diseases 5(5): e1143.

40. Nalugwa, A., F. Nuwaha, E. M. Tukahebwa and A. Olsen (2015). "Single Versus Double Dose Praziquantel Comparison on Efficacy and Schistosoma mansoni Re-Infection in Preschool-Age Children in Uganda: A Randomized Controlled Trial." Plos Neglected Tropical Diseases 9(5): e0003796-e0003796.

41. Namwanje, H., N. B. Kabatereine and A. Olsen (2011). "The acceptability and safety of praziquantel alone and in combination with mebendazole in the treatment of Schistosoma mansoni and soiltransmitted helminthiasis in children aged 1-4 years in Uganda." Parasitology 138(12): 1586-1592.

42. Nandha, B., C. Sadanandane, P. Jambulingam and P. K. Das (2007). "Delivery strategy of mass annual single dose DEC administration to eliminate lymphatic filariasis in the urban areas of Pondicherry, South India: 5 years of experience." Filaria Journal 6(1): 7.

43. Navaratnam, A. M. D., J. C. Sousa-Figueiredo, J. R. Stothard, N. B. Kabatereine, A. Fenwick and M. J. Mutumba-Nakalembe (2012). "Efficacy of praziquantel syrup versus crushed praziquantel tablets in the treatment of intestinal schistosomiasis in Ugandan preschool children, with observation on compliance and safety." Transactions Of The Royal Society Of Tropical Medicine And Hygiene 106(7): 400-407.

44. Ochodo, E. A., B. Spek, J. B. Reitsma, L. Van Lieshout, K. Polman, P. Lamberton, P. M. Bossuyt and M. M. Leeflang (2012). "Rapid screening and diagnostic tests for human schistosomiasis in endemic areas." Cochrane Database of Systematic Reviews(2). 
45. Olliaro, P. L., M. Vaillant, D. J. Hayes, A. Montresor and L. Chitsulo (2013). "Practical dosing of praziquantel for schistosomiasis in preschool-aged children." Tropical Medicine \& International Health 18(9): 1085-1089.

46. Olveda, D. U., D. P. McManus and A. G. Ross (2016). "Mass drug administration and the global control of schistosomiasis: successes, limitations and clinical outcomes." Current opinion in infectious diseases 29(6): 595-608.

47. Olveda, R. M., L. P. Acosta, V. Tallo, P. I. Baltazar, J. L. S. Lesiguez, G. G. Estanislao, E. B. Ayaso, D. B. S. Monterde, A. Ida, N. Watson, E. A. McDonald, H. W. Wu, J. D. Kurtis and J. F. Friedman (2016). "Efficacy and safety of praziquantel for the treatment of human schistosomiasis during pregnancy: a phase 2, randomised, double-blind, placebo-controlled trial." The Lancet Infectious Diseases 16(2): 199-208.

48. Osakunor, D. N. M., T. Mduluza, N. Midzi, M. Chase-Topping, M. J. Mutsaka-Makuvaza, T. Chimponda, E. Eyoh, T. Mduluza, L. T. Pfavayi, W. M. Wami, S. A. Amanfo, J. Murray, C. Tshuma, M. E. J. Woolhouse and F. Mutapi (2018). "Dynamics of paediatric urogenital schistosome infection, morbidity and treatment: a longitudinal study among preschool children in Zimbabwe." BMJ Global Health 3(2).

49. Osakunor, D. N. M., M. E. J. Woolhouse and F. Mutapi (2018). "Paediatric schistosomiasis: What we know and what we need to know." PLOS Neglected Tropical Diseases 12(2): e0006144.

50. Peterson, J., J. R. Atwood and B. Yates (2002). "Key elements for church-based health promotion programs: outcome-based literature review." Public Health Nursing 19(6): 401-411.

51. Ross, A. G., R. M. Olveda, D. Chy, D. U. Olveda, Y. Li, D. A. Harn, D. J. Gray, D. P. McManus, V. Tallo and T. N. Chau (2014). "Can mass drug administration lead to the sustainable control of schistosomiasis?" The Journal of infectious diseases 211(2): 283-289.

52. Sousa-Figueiredo, J., M. Betson, A. Atuhaire, M. Arinaitwe and A. Navaratnam (2012). "Performance and Safety of Praziquantel for Treatment of Intestinal Schistosomiasis in Infants and Preschool Children." PLOS Neglected Tropical Diseases 6(10): e1864.

53. Sousa-Figueiredo, J. C., M. Betson, N. B. Kabatereine and J. R. Stothard (2013). "The urine circulating cathodic antigen (CCA) dipstick: a valid substitute for microscopy for mapping and point-of-care diagnosis of intestinal schistosomiasis." PLoS neglected tropical diseases 7(1): e2008.

54. Sousa-Figueiredo, J. C., M. Betson and J. R. Stothard (2012). "Treatment of schistosomiasis in African infants and preschool-aged children: downward extension and biometric optimization of the current praziquantel dose pole." International health 4(2): 95-102.

55. Sousa-Figueiredo, J. C., J. Pleasant, M. Day, M. Betson, D. Rollinson, A. Montresor, F. Kazibwe, N. B. Kabatereine and J. R. Stothard (2010). "Treatment of intestinal schistosomiasis in Ugandan preschool children: best diagnosis, treatment efficacy and side-effects, and an extended praziquantel dosing pole." International Health 2(2): 103-113.

56. Stothard, J. R., J. C. Sousa-Figueiredo, M. Betson, A. Bustinduy and J. Reinhard-Rupp (2013). "Schistosomiasis in African infants and preschool children: let them now be treated!" Trends in 
Parasitology 29(4): 197-205.

57. Stothard, J. R., J. C. Sousa-Figueiredo, M. Betson, H. K. Green, E. Y. W. Seto, A. Garba, M. Sacko, F. Mutapi, S. Vaz Nery, M. A. Amin, M. Mutumba-Nakalembe, A. Navaratnam, A. Fenwick, N. B.

Kabatereine, A. F. Gabrielli and A. Montresor (2011). "Closing the praziquantel treatment gap: new steps in epidemiological monitoring and control of schistosomiasis in African infants and preschoolaged children." Parasitology 138(12): 1593-1606.

58. Thong, M. Y., Y. J. Manrique and K. J. Steadman (2018). "Drug loss while crushing tablets: Comparison of 24 tablet crushing devices." PLOS ONE 13(3): e0193683.

59. Turner, H. C., J. Toor, T. Déirdre Hollingsworth and R. M. Anderson (2017). "Economic evaluations of mass drug administration: The importance of economies of scale and scope." Clinical Infectious Diseases 66(8): 1298-1303.

60. Utzinger, J., S. L. Becker, L. van Lieshout, G. J. van Dam and S. Knopp (2015). "New diagnostic tools in schistosomiasis." Clinical Microbiology and Infection 21(6): 529-542.

61. Wami, W. M., N. Nausch, N. Midzi, R. Gwisai, T. Mduluza, M. E. J. Woolhouse and F. Mutapi (2016). "Comparative Assessment of Health Benefits of Praziquantel Treatment of Urogenital Schistosomiasis in Preschool and Primary School-Aged Children." Biomed Research International 2016: $9162631-9162631$.

62. WHO (2006). Preventive chemotherapy in human helminthiasis. Manual on preventive chemotherapy.

63. WHO (2017). "Schistosomiasis and soil-transmitted helminthiases: number of people treated in 2016." Wkly Epidemiol Rec. 749-760(49): 749-760.

64. WHO. (2018). "Schistosomiaisis Control and preventive chemotherapy." 2018, from http://www.who.int/schistosomiasis/strategy/en/.

\section{Tables}

Tables 1 and 2 are not available with this version.

\section{Figures}




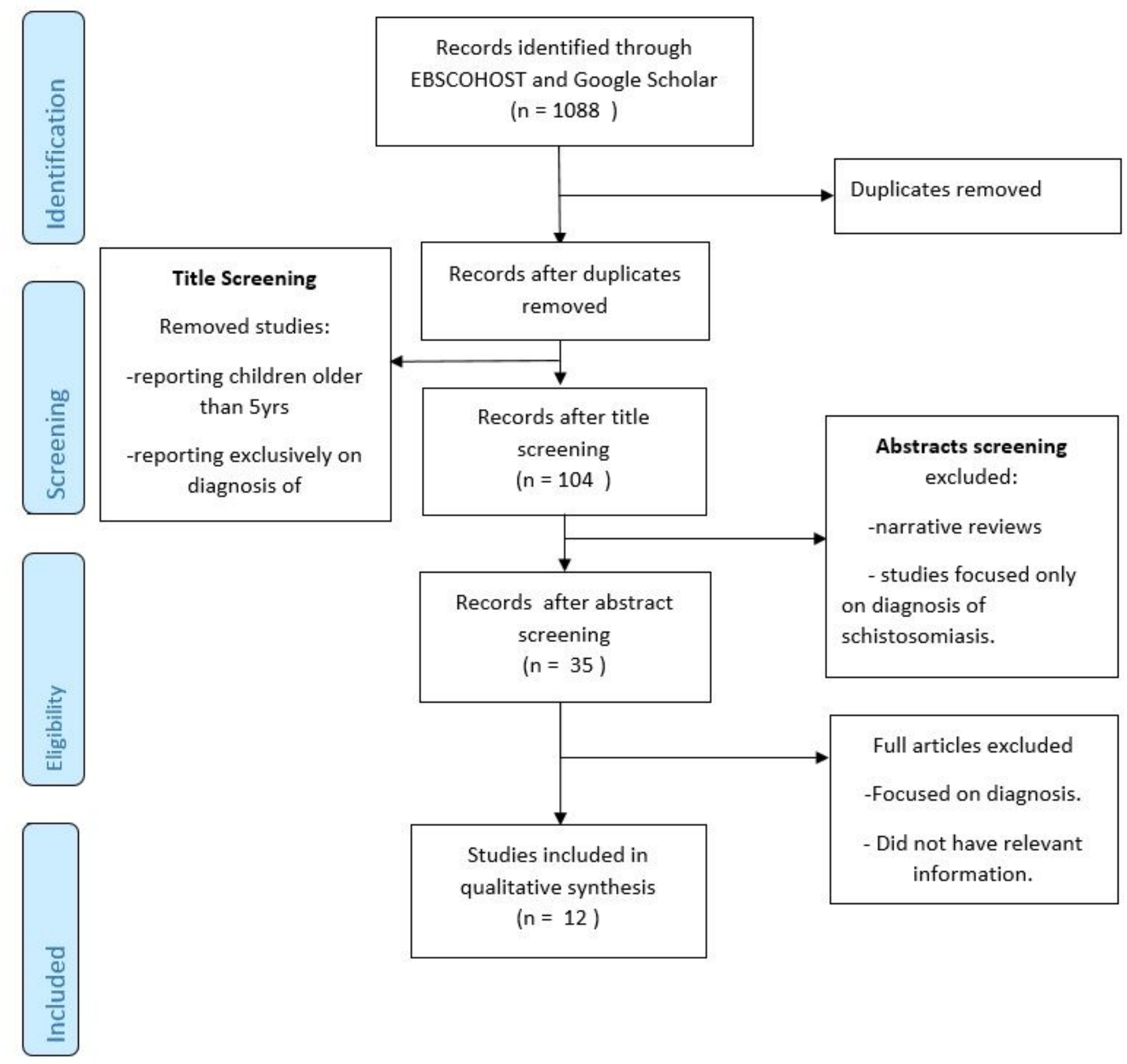

\section{Figure 1}

PRISMA chart describing the search process for selecting final papers for the review 


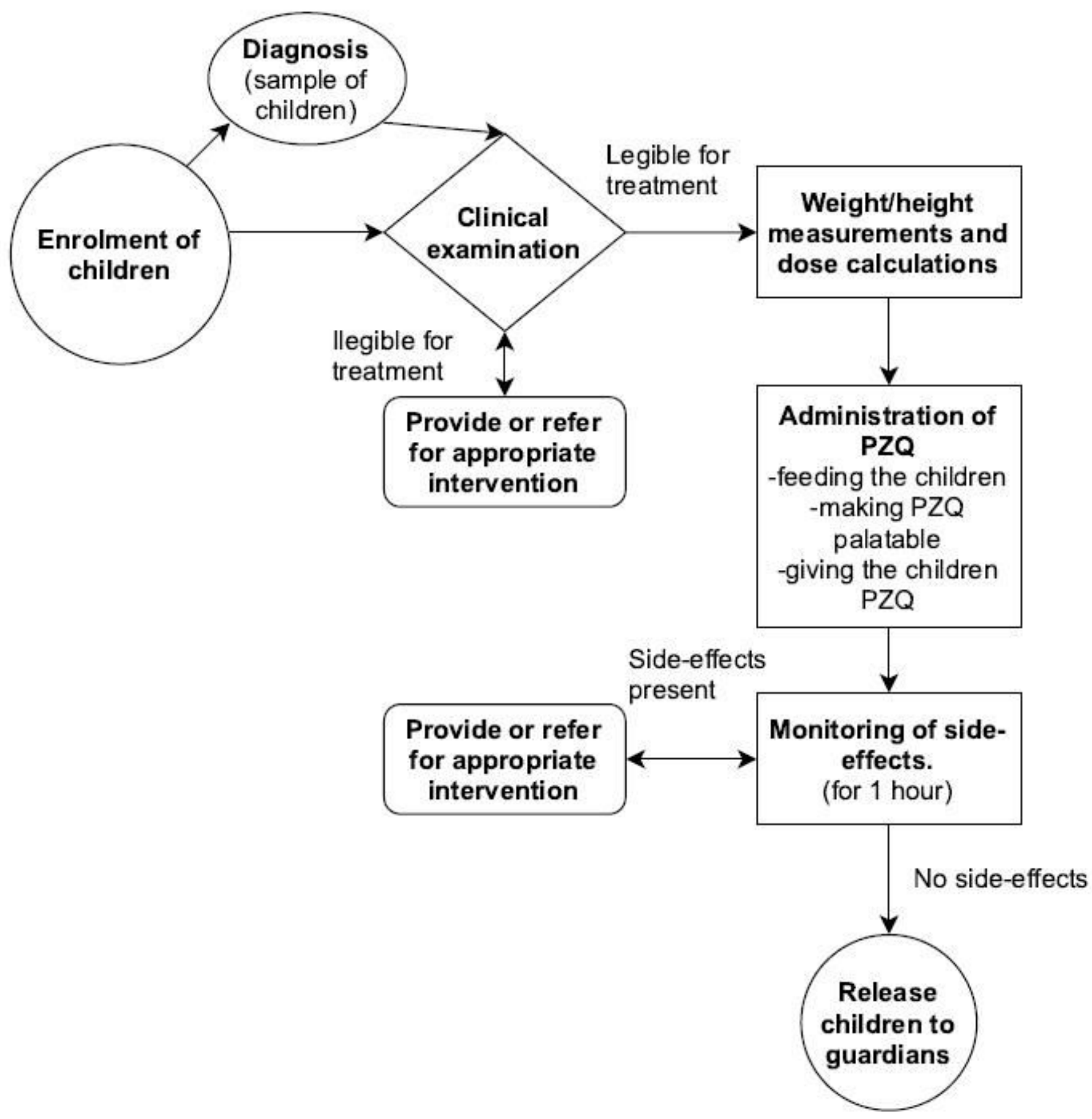

Figure 2

Treatment process flow diagram for a schistosomiasis control MDA program for children aged five years old and below.

\section{Supplementary Files}

This is a list of supplementary files associated with this preprint. Click to download.

- PRISMAChecklist.doc 